\title{
Neuronal Functions of ESCRTs
}

\author{
Jin-A Lee ${ }^{1 *}$ and Fen-Biao Gao ${ }^{2}$ \\ ${ }^{1}$ Department of Biotechnology, College of Life Science and Nano Technology, Hannam University, Dajeon 305-811, Korea, \\ ${ }^{2}$ Department of Neurology, University of Massachusetts Medical School, Worcester, Massachusetts, 01605 USA
}

The endosomal sorting complexes required for transport (ESCRTs) regulate protein trafficking from endosomes to lysosomes. Recent studies have shown that ESCRTs are involved in various cellular processes, including membrane scission, microRNA function, viral budding, and the autophagy pathway in many tissues, including the nervous system. Indeed, dysfunctional ESCRTs are associated with neurodegeneration. However, it remains largely elusive how ESCRTs act in post-mitotic neurons, a highly specialized cell type that requires dynamic changes in neuronal structures and signaling for proper function. This review focuses on our current understandings of the functions of ESCRTs in neuronal morphology, synaptic plasticity, and neurodegenerative diseases.

Key words: autophagy, dendrites, endocytosis, ESCRTs, lysosome, neurodegenerative diseases

\section{INTRODUCTION}

Multivesicular bodies (MVBs) are involved in the delivery of ubiquitinated cargo proteins to lysosomes for degradation. The endosomal sorting complexes required for transport (ESCRTs) regulate the MVB pathway through MVB biogenesis and cargo sorting into lysosomes. The ESCRTs, first identified in yeast, are composed of four different protein complexes called ESCRT-0, -I, -II, -III and several accessory proteins. Each complex has multiple subunits and is sequentially and temporally recruited into the endosomal membrane during endocytic trafficking of cargo proteins. Ubiquitinated cargo proteins are recognized and captured by the ESCRT machinery $[1,2]$. Emerging evidence suggests that different ubiquitin binding subunits regulate cargo sorting, membrane deformation, and final vesicle scission [1]. Among the ESCRT complexes, ESCRT-III is particularly involved in the invagination of cargo proteins into MVB vesicles. In the

\footnotetext{
Received January 17,2012, Accepted January 30, 2012

* To whom correspondence should be addressed.

TEL: 82-42-629-8785, FAX: 82-42-629-8769

e-mail: leeja@hnu.kr
}

final step, MVBs are fused with lysosomes for cargo protein degradation. This machinery is functionally conserved from yeast to humans. ESCRT functions in the endo-lysosomal pathway have been extensively studied for the last 10 15 years using genetic or biochemical approaches with in vitro cell culture and in vivo models. Although the ESCRT function in regulating cell signaling through receptor downregulation has been well established, recent studies have suggested that ESCRTs are also involved in other cellular processes, including viral budding, cytokinesis, tumor suppression, autophagy, and microRNA function [2]. Moreover, ESCRT dysfunction is associated with several human diseases such as cancer and neurodegenerative diseases [3].

Neurons have specialized structures such as axons and dendrites that perform different functions. Dendrites receive and process information from axonal terminals that release neurotransmitters for proper neuronal signaling. This dynamic signaling of axons and dendrites requires the correct delivery of proper molecules to the target region depending on the tight regulation of protein sorting and degradation. Therefore, the regulation of neuronal cell signaling via the endo-lysosomal pathway in specialized regions of the dendritic and axonal membranes and the neuronal cell body is essential for neuronal cell survival, growth, synapse
Copyright @ Experimental Neurobiology 2012. www.enjournal.org
This is an Open Access article distributed under the terms of the Creative Commons Attribution Non-Commercial License (http://creativecommons.org/licenses/by-nc/3.0) which permits unrestricted non-commercial use, distribution, and reproduction in any medium, provided the original work is properly cited. 
formation, and various synaptic functions [4]. Indeed, dysfunction of the endocytic or lysosomal pathway is associated with several neurodegenerative diseases such as Alzheimer's, Huntington's, and Parkinson's diseases [5]. The ESCRT proteins that regulate the key sorting step of cargo proteins in the endosomal pathway are widely expressed in the central nervous system and their dysfunction can contribute to neurodegeneration [6, 7]. Additionally, the ESCRT machinery has been recently implicated in neuronal morphogenesis and synaptic function [6, 8-11]. However, the exact function of ESCRTs in neurons remains to be fully identified. In this review, we will briefly summarize the composition of the ESCRT complexes and focus on ESCRT functions related to neuronal physiology and pathology. The readers are referred to several excellent reviews for more in-depth knowledge about ESCRT biology $[1,3,12,13]$.

\section{THE ESCRT COMPLEXES}

The ESCRTs were first identified as class E vacuolar protein sorting (Vps) proteins in yeast. Several genetic and biochemical studies have shown that loss of these genes causes the formation of abnormal MVBs lacking internal vesicles and the accumulation of cargo proteins, suggesting a role for ESCRTs in MVB biogenesis and cargo sorting [14]. The class E Vps proteins are functionally conserved from yeast to humans, indicating that the MVB pathway is universal. Interestingly, some Vps proteins in yeast have multiple mammalian paralogs, although the specific function of each paralog is currently being investigated under various biological contexts $[15,16]$. The ESCRTs from four heteromeric protein complexes named ESCRT-0, -I, -II, and -III are involved in cargo recognition, cargo-sorting, and lipid membranedeformation by sequential recruitment and recycling of each complex into the endosomal membrane from the cytosol [1-3].

\section{ESCRT-0}

ESCRT-0 is composed of two subunits, hepatocyte growth factor regulated tyrosine kinase substrate (Hrs, Vps27p in yeast) and signal transducing adaptor molecule 1/2 (STAM, Hselp in yeast), which can interact with ubiquitin. Among these components, Hrs controls the specificity and membrane recruitment of ESCRT-0 at the endosome by binding phosphatidylinositol 3-phosphate [1, 3]. Therefore, ESCRT-0 triggers the initiation of MVB-dependent cargo sorting by recruiting ESCRT-I into the endosomal membrane in yeast and metazoans $[1,3,17]$.

\section{ESCRT-I}

Among ESCRTs, ESCRT-I was first characterized in yeast, and it has four components, including Tsg101 (Vps23 in yeast), Vps28, Vps37, and recently identified Mvb12 [18, 19]. ESCRT-I binds to ESCRT-II and ESCRT-0 for further recruitment of ESCRT subunits and cargo proteins into endosomal membranes through the ubiquitin E2 variant domain of ESCRT-I subunits and the PTAP-like motif of ESCRT-0 subunits [17, 20].

\section{ESCRT-II}

ESCRT-II, which consists of Vps36, one Vps22, and two Vps25 subunits, has eight winged-helix repeats [21]. The GRAM-like ubiquitin binding Eap45 domain in Vps36 is involved in binding to ubiquitin and 3-phosphorylated phosphoinositides in the endosomal membrane and also connects to Vps28 of ESCRT-I in yeast, although the link between ESCRT-I and ESCRT-II in mammals has yet to be characterized [22]. Vps25 contributes to initiating formation of the ESCRT-III complex through interaction with Vps20, which is an ESCRT-III subunit [2].

\section{ESCRT-III}

Unlike other ESCRT complexes, ESCRT-III consists of four highly charged subunits such as Vps20, Snf7, Vps24, and Vps2, and it does not form a stable cytosolic complex [23]. Interestingly, monomers of each ESCRT subunit are inactive due to their interaction with the $\mathrm{N}$-terminus and the autoinhibitory C-terminus of the ESCRT-III component in the cytosol [13, 24, 25]. Conformational changes in the ESCRT-III monomer precede the assembly of high-order multimers on membranes via interactions with other ESCRT-III subunits. The assembly of ESCRT-III begins with an interaction between Vps25 and Vps20, which recruits Snf7. Snf7, as the most abundant protein among ESCRT-III proteins, forms filamentous structures by homo-oligomerization leading to recruitment of Vps24Vps2. Then, Vps24-Vps2 recruits AAA ATPase Vps4 into the endosomal membrane to disassemble the ESCRT-III complex from the membrane $[26,27]$. Brol/Alix can also regulate the Snf7 nucleation process in an ESCRT-II-independent manner. The assembly of ESCRT-III is involved in driving membrane deformation [27]. Additionally, accessory proteins such as Ist1, Doa4-independent degradation-2 (Did2), and Vps twentyassociated 1 (Vta1) regulate Vps4 function for the proper recycling of ESCRT-III subunits [28-30].

\section{ESCRTS IN POST-MITOTIC NEURONS}

A significant amount of membrane trafficking and cargo protein sorting occurs in specialized compartments in post-mitotic neurons during neuronal differentiation and function. In addition, 


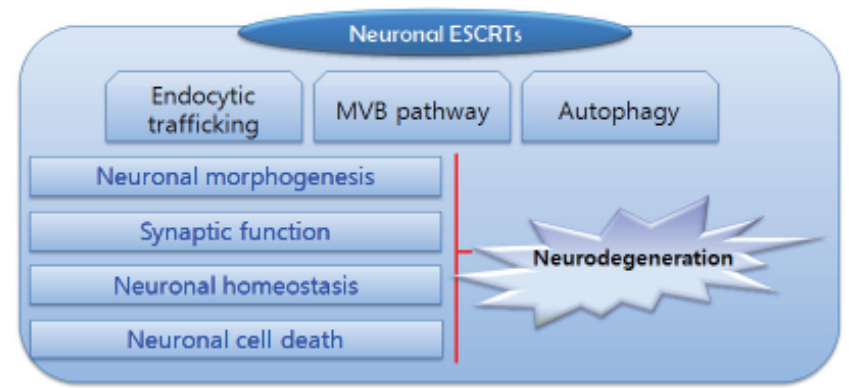

Fig. 1. Neuronal ESCRT as a multiplayer in post-mitotic neurons. ESCRTs regulate endocytic trafficking, membrane deformation, or the autophagy pathway in post-mitotic neurons, although they are involved in other unknown cellular processes. Neuronal ESCRTs control neuronal morphogenesis such as axonal and dendritic growth, synaptic growth, and may affect neuronal signaling at synaptic sites by down-regulating signaling molecules in response to various synaptic activities. Furthermore, neuronal homeostasis is regulated by controlling the degradation of cargo proteins or cytosolic components via the ESCRT involved in the autolysosomal pathway. Interestingly, the ESCRT may directly or indirectly contribute to neuronal cell death. Some ESCRT components may be linked to the cell death pathway in a direct manner. Dysfunction of ESCRTs can cause neurodegeneration leading to neuronal cell death.

endosomal trafficking and lysosomal functions in the central nervous system play important roles in neuronal cell survival and proper neuronal signaling. Therefore, these pathways should be tightly regulated. ESCRTs are key components that regulate sorting and degradation of cargo proteins and membrane deformation. Thus, neuronal ESCRTs must be important players in various neuronal functions (Fig. 1).

\section{ESCRTs in neuronal morphogenesis}

Neurons have highly polarized structures such as axons and dendrites, which are striking features of a mature nervous system. During axonal or dendritic growth and arborization, many membrane proteins such as TrkB or Eph receptors in response to proper signals may be sorted into the endo-lysosomal pathway to regulate neuronal morphogenesis $[31,32]$. Indeed, loss of Shrub, which is a major component of ESCRT-III in Drosphila PNS neurons, results in a dramatic decrease in the dendritic field and an increase in dendritic branching in a cell-autonomous manner, indicating the role of ESCRT-III in the control of neuronal morphogenesis [8]. Furthermore, dysfunction of ESCRT-III either by loss of mammalian Snf7-2, which is a homolog of Drosophila Shrub, or by overexpression of frontotemporal dementia (FTD)-linked CHMP2B mutant protein in cultured mature cortical neurons causes dendritic retraction, suggesting that neuronal ESCRT-III is involved in the maintenance of dendritic morphology $[6,33]$. Interestingly, a recent study suggested that neuronal CHMP2B also regulates dendritic spine maturation [9]. FTD-linked CHMP2B mutant proteins induce a reduction in large mushroom-shaped spines, and the loss of CHMP2B in cultured hippocampal neurons also shows similar abnormal spine morphology. Therefore, neuronal ESCRTs induce neuronal morphological changes possibly by endocytic sorting of cargo proteins involved in neuronal growth and maintenance of neuronal morphology. Whether ESCRTs have other functions in neuronal morphogenesis independent of the endo-lysosomal pathway remains to be seen. The exact function of ESCRTs in neuronal morphogenesis should be also evaluated in an in vivo mouse model.

\section{ESCRTs in endosomal trafficking at synapses in neurons}

Neurons require highly dynamic neuronal signaling in axons and dendrites. The ESCRTs are localized at axons and dendrites, indicating their possible roles in endosomal trafficking at presynaptic and postsynaptic sites $[6,8]$. An increasing body of evidence shows that ESCRTs together with other regulators in the endocytic pathway regulate cargo protein sorting at synapses. Synaptic proteins at axonal terminals undergo a dynamic recycling and degradation to regulate presynaptic growth and neurotransmitter release. Interestingly, a recent study suggested that the ESCRT complex mediates synaptic vesicle protein sorting in sky (Rab 32 GAP in Drosophila) mutants where synaptic vesicles are recycled excessively via endosomes [10]. Besides protein sorting in the sky mutant, artificially generated ubiquitinated synaptic vesicle proteins are degraded in an ESCRTdependent manner, suggesting that ESCRTs might control the nature and steady-state level of proteins involved in synaptic vesicle recycling and neuronal signaling. Another example of neuronal ESCRTs in the nerve terminal is the ESCRT-dependent presynaptic endosomal trafficking pathway to control synaptic growth signals. ESCRTs together with SNX16 regulate synaptic growth at the interface between endosomal compartments [11].

The surface level of several neurotransmitter receptors or membrane proteins at post-synaptic sites is crucial for temporal and spatial neuronal signaling, and this can be regulated by the endosomal trafficking pathway. Indeed, loss of Snf7s in human neurons causes the accumulation of neurotransmitter receptors such as epidermal growth factor receptor (EGFR), GluR1, NR1, and TrkA or membrane proteins such as APP [16]. Additionally, recent data show that the GPCR interacting scaffold protein, which is a brain-specific scaffold protein, retards TSG101-dependent lysosomal degradation of neurotransmitter receptors through its interaction with TSG101 $[34,35]$. However, the mechanism of how synaptic cargo proteins are specifically recognized by ESCRTs and are sorted into proper endosomal compartments 
remains to be elucidated. Moreover, the specific function of each ESCRT complex during trafficking and degradation of synaptic cargo proteins in response to various synaptic activities needs to be investigated. Further characterization of neuronal ESCRTs in synapses will help us to understand the functions of ESCRTs in synaptic plasticity.

\section{ESCRTs in neuronal cell death}

Endocytosis associated with the autophagy pathway increases massively during neuronal cell death at the early developmental stages, indicating that activation of the endo-lysosomal pathway is required for some types of cell death [36]. Alix, first characterized as an ALG-2 interacting protein, binds to TSG101 and Snf7-2 (also known as CHMP4b) [37-39]. Alix overexpression induces apoptosis in cultured cerebellar granule neurons and in the developing chick spinal cord, suggesting that ESCRT-associated Alix might cause neuronal cell death [36]. Interestingly, disrupting the interaction between Alix and ESCRTs inhibits neuronal cell death, supporting the idea that the Alix/ESCRT complex could contribute to neuronal cell death. Further studies are needed to uncover the detailed mechanism by which Alix/ESCRT induces neuronal cell death $[40,41]$.

\section{ESCRTs in neurodegeneration}

Several neurodegenerative diseases such as Alzheimer's disease, Huntington's disease, and Parkinson's disease are associated with abnormal endo-lysosomal functions [42]. Among endo-lysosomal proteins, ESCRT dysfunction could also lead to neurodegeneration and it is associated with some neurodegenerative diseases. The mutation in CHMP2B, a key component of ESCRT-III, was firstly identified in frontotemporal dementia linked to chromosome-3 (FTD-3), raising the possibility that dysfunction of ESCRT is associated with the pathogenesis of FTD [33]. This was supported by the finding that the FTDlinked mutant CHMP2B protein, a key component of ESCRTIII, causes dendritic retraction and accumulation of ubiquitinated proteins and autophagosomes leading to neuronal cell loss in cultured cortical neurons $[6,33,43]$. Interestingly, several mutations in CHMP2B have been subsequently identified in patients with FTD, FTD-MND (Frontotemporal dementia with motor neuron diseases), or Amyotrophic lateral sclerosis (ALS) [44-46]. Moreover, strong CHMP2B immunopositivity during granulovacuolar degeneration of neurons has been detected in patients with Alzheimer's disease, indicating that CHMP2B is associated with a broad spectrum of neurodegenerative diseases [47]. Additionally, loss of Hrs, which is a core component of ESCRT-I in the central nervous system of a mice model, causes accumulation of ubiquitinated protein, neurodegeneration, and impaired learning ability and locomotor activity [7]. These studies support the essential role of ESCRTs in neuronal cell survival and protein homeostasis.

There are other examples of ESCRT dysfunction associated neurodegeneration. Spongiform neurodegeneration-associated E3 ligase Mahogunin ubiquitylates TSG101 and regulates endosomal trafficking. Loss of Mahogunin in an animal model results in abnormal regulation of TSG101 leading to spongiform neurodegeneration $[48,49]$. The dysfunction of accessory proteins related to the ESCRT complex is also associated with neurodegeneration. The ESCRT-III-associated endosomal protein CHMP1B interacts with the hereditary spastic paraplegia protein (HSP) spastin, increasing the possibility of a contribution by CHMP1B in the pathogenesis of HSP [50].

If so, how does ESCRT dysfunction lead to neurodegeneration? ESCRT dysfunction disrupts endosomal trafficking and degradation of cargo proteins, which contribute to cellular toxicity and neurodegeneration. Besides endo-lysosomal dysfunction, ESCRT dysfunction causes accumulation of autophagosomes probably due to the inhibition of the maturation of autophagosomes to autolysosomes $[6,33,51]$. Inhibiting the accumulation of autophagosomes delays ESCRT dysfunctioninduced neurodegeneration, suggesting that autophagy could partially contribute to neuronal cell loss under some circumstances [52]. Therefore, understanding of how ESCRTs are involved in endosomal traffic and the fusion or maturation of autophagosomes will no doubt lead to better understanding of the molecular pathogenic events in several neurodegenerative diseases associated with ESCRTs.

\section{CONCLUSION \& PERSPECTIVES}

Much has been learned about the ESCRT functions in various biological systems. Neuronal ESCRTs function as key endocytic regulators of cargo protein sorting between endosomal compartments and as a modifier of membrane dynamics (membrane deformation and scission). Additionally, neuronal ESCRTs are required for neuronal homeostasis by controlling maturation of autophagosomes and degradation of cargo proteins in the auto-lysosomal pathway (Fig. 1). Despite our current knowledge about ESCRT functions, many questions still remain. For instance, the spatial and temporal regulation and specificity of ESCRTs under dynamic neuronal signaling need to be further elucidated.

Unlike their yeast counterparts, mammalian ESCRTs have multiple isoforms. Interestingly, recent studies have shown that the 
distinct ESCRT complex may have distinct functional specificity $[15,16,53]$. Whether each isoform of the neuronal ESCRT complex has specificity in its expression and localization in axons or dendrites, in subunit composition, or in cargo selection should be further elucidated. All of these studies will provide a further understanding of the neuronal functions of ESCRTs and may lead to the development of therapeutic intervention for neurodegenerative diseases associated with ESCRTs.

\section{ACKNOWLEDGEMENTS}

We apologize to colleagues whose work could not be cited due to space limitations. JAL was supported by a grant from the Korean Health Technology R\&D Project, Ministry for Health, Welfare, \& Family Affairs, Republic of Korea (A100488) and by the Basic Science Research Program through the National Research Foundation of Korea (NRF) funded by the Ministry of Education, Science, and Technology (2010-0010824). FBG is supported by the National Institutes of Health (RO1NS057553).

\section{REFERENCES}

1. Raiborg C, Stenmark H (2009) The ESCRT machinery in endosomal sorting of ubiquitylated membrane proteins. Nature 458:445-452

2. Henne WM, Buchkovich NJ, Emr SD (2011) The ESCRT pathway. Dev Cell 21:77-91

3. Saksena S, Emr SD (2009) ESCRTs and human disease. Biochem Soc Trans 37:167-172

4. Bradke F, Dotti CG (1998) Membrane traffic in polarized neurons. Biochim Biophys Acta 1404:245-258

5. Nixon RA, Yang DS, Lee JH (2008) Neurodegenerative lysosomal disorders: a continuum from development to late age. Autophagy 4:590-599

6. Lee JA, Beigneux A, Ahmad ST, Young SG, Gao FB (2007) ESCRT-III dysfunction causes autophagosome accumulation and neurodegeneration. Curr Biol 17:1561-1567

7. Tamai K, Toyoshima M, Tanaka N, Yamamoto N, Owada Y, Kiyonari H, Murata K, Ueno Y, Ono M, Shimosegawa T, Yaegashi N, Watanabe M, Sugamura K (2008) Loss of hrs in the central nervous system causes accumulation of ubiquitinated proteins and neurodegeneration. Am J Pathol 173:1806-1817

8. Sweeney NT, Brenman JE, Jan YN, Gao FB (2006) The coiledcoil protein shrub controls neuronal morphogenesis in Drosophila. Curr Biol 16:1006-1011

9. Belly A, Bodon G, Blot B, Bouron A, Sadoul R, Goldberg Y
(2010) CHMP2B mutants linked to frontotemporal dementia impair maturation of dendritic spines. J Cell Sci 123:29432954

10. Uytterhoeven V, Kuenen S, Kasprowicz J, Miskiewicz K, Verstreken P (2011) Loss of skywalker reveals synaptic endosomes as sorting stations for synaptic vesicle proteins. Cell 145:117-132.

11. Rodal AA, Blunk AD, Akbergenova Y, Jorquera RA, Buhl LK, Littleton JT (2011) A presynaptic endosomal trafficking pathway controls synaptic growth signaling. J Cell Biol 193:201-217

12. Hurley JH, Im YJ, Lee HH, Ren X, Wollert T, Yang D (2009) Piecing together the ESCRTs. Biochem Soc Trans 37:161-166

13. Williams RL, Urbé S (2007) The emerging shape of the ESCRT machinery. Nat Rev Mol Cell Biol 8:355-368

14. Babst M (2005) A protein's final ESCRT. Traffic 6:2-9

15. Stefani F, Zhang L, Taylor S, Donovan J, Rollinson S, Doyotte A, Brownhill K, Bennion J, Pickering-Brown S, Woodman P (2011) UBAP1 is a component of an endosome-specific ESCRT-I complex that is essential for MVB sorting. Curr Biol 21:1245-1250

16. Lee JA, Liu L, Javier R, Kreitzer AC, Delaloy C, Gao FB (2011) ESCRT-III subunits Snf7-1 and Snf7-2 differentially regulate transmembrane cargos in hESC-derived human neurons. Mol Brain 4:37

17. Katzmann DJ, Stefan CJ, Babst M, Emr SD (2003) Vps27 recruits ESCRT machinery to endosomes during MVB sorting. J Cell Biol 162:413-423

18. Chu T, Sun J, Saksena S, Emr SD (2006) New component of ESCRT-I regulates endosomal sorting complex assembly. J Cell Biol 175:815-823

19. Morita E, Sandrin V, Alam SL, Eckert DM, Gygi SP, Sundquist WI (2007) Identification of human MVB12 proteins as ESCRT-I subunits that function in HIV budding. Cell Host Microbe 2:41-53

20. Kostelansky MS, Sun J, Lee S, Kim J, Ghirlando R, Hierro A, Emr SD, Hurley JH (2006) Structural and functional organization of the ESCRT-I trafficking complex. Cell 125:113-126

21. Hierro A, Sun J, Rusnak AS, Kim J, Prag G, Emr SD, Hurley JH (2004) Structure of the ESCRT-II endosomal trafficking complex. Nature 431:221-225

22. Teo H, Gill DJ, Sun J, Perisic O, Veprintsev DB, Vallis Y, Emr SD, Williams RL (2006) ESCRT-I core and ESCRT-II GLUE domain structures reveal role for GLUE in linking to ESCRT-I and membranes. Cell 125:99-111

23. Babst M, Katzmann DJ, Estepa-Sabal EJ, Meerloo T, Emr SD 
(2002) Escrt-III: an endosome-associated heterooligomeric protein complex required for mvb sorting. Dev Cell 3:271-282

24. Shim S, Kimpler LA, Hanson PI (2007) Structure/function analysis of four core ESCRT-III proteins reveals common regulatory role for extreme C-terminal domain. Traffic 8:1068-1079

25. Lata S, Roessle M, Solomons J, Jamin M, Gottlinger HG, Svergun DI, Weissenhorn W (2008) Structural basis for autoinhibition of ESCRT-III CHMP3. J Mol Biol 378:818-827

26. Teis D, Saksena S, Emr SD (2008) Ordered assembly of the ESCRT-III complex on endosomes is required to sequester cargo during MVB formation. Dev Cell 15:578-589

27. Saksena S, Wahlman J, Teis D, Johnson AE, Emr SD (2009) Functional reconstitution of ESCRT-III assembly and disassembly. Cell 136:97-109

28. Azmi I, Davies B, Dimaano C, Payne J, Eckert D, Babst M, Katzmann DJ (2006) Recycling of ESCRTs by the AAAATPase Vps4 is regulated by a conserved VSL region in Vtal. J Cell Biol 172:705-717

29. Azmi IF, Davies BA, Xiao J, Babst M, Xu Z, Katzmann DJ (2008) ESCRT-III family members stimulate Vps4 ATPase activity directly or via Vta1. Dev Cell 14:50-61

30. Yu Z, Gonciarz MD, Sundquist WI, Hill CP, Jensen GJ (2008) Cryo-EM structure of dodecameric Vps4p and its 2:1 complex with Vtalp. J Mol Biol 377:364-377

31. Henkemeyer M, Itkis OS, Ngo M, Hickmott PW, Ethell IM (2003) Multiple EphB receptor tyrosine kinases shape dendritic spines in the hippocampus. J Cell Biol 163:13131326

32. Passafaro M, Nakagawa T, Sala C, Sheng M (2003) Induction of dendritic spines by an extracellular domain of AMPA receptor subunit GluR2. Nature 424:677-681

33. Skibinski G, Parkinson NJ, Brown JM, Chakrabarti L, Lloyd SL, Hummerich H, Nielsen JE, Hodges JR, Spillantini MG, Thusgaard T, Brandner S, Brun A, Rossor MN, Gade A, Johannsen P, Sørensen SA, Gydesen S, Fisher EM, Collinge J (2005) Mutations in the endosomal ESCRTIII-complex subunit CHMP2B in frontotemporal dementia. Nat Genet 37:806-808

34. Kantamneni S, Holman D, Wilkinson KA, Corrêa SA, Feligioni M, Ogden S, Fraser W, Nishimune A, Henley JM (2008) GISP binding to TSG101 increases GABA receptor stability by down-regulating ESCRT-mediated lysosomal degradation. J Neurochem 107:86-95

35. Kantamneni S, Holman D, Wilkinson KA, Nishimune A, Henley JM (2009) GISP increases neurotransmitter receptor stability by down-regulating ESCRT-mediated lysosomal degradation. Neurosci Lett 452:106-110

36. Mahul-Mellier AL, Hemming FJ, Blot B, Fraboulet S, Sadoul R (2006) Alix, making a link between apoptosis-linked gene2 , the endosomal sorting complexes required for transport, and neuronal death in vivo. J Neurosci 26:542-549

37. Martin-Serrano J, Yarovoy A, Perez-Caballero D, Bieniasz PD (2003) Divergent retroviral late-budding domains recruit vacuolar protein sorting factors by using alternative adaptor proteins. Proc Natl Acad Sci U S A 100:12414-12419

38. Strack B, Calistri A, Craig S, Popova E, Göttlinger HG (2003) AIP1/ALIX is a binding partner for HIV-1 p6 and EIAV p9 functioning in virus budding. Cell 114:689-699

39. von Schwedler UK, Stuchell M, Müller B, Ward DM, Chung HY, Morita E, Wang HE, Davis T, He GP, Cimbora DM, Scott A, Kräusslich HG, Kaplan J, Morham SG, Sundquist WI (2003) The protein network of HIV budding. Cell 114:701713

40. Blum D, Hemming FJ, Galas MC, Torch S, Cuvelier L, Schiffmann SN, Sadoul R (2004) Increased Alix (apoptosislinked gene-2 interacting protein X) immunoreactivity in the degenerating striatum of rats chronically treated by 3-nitropropionic acid. Neurosci Lett 368:309-313

41. Hemming FJ, Fraboulet S, Blot B, Sadoul R (2004) Early increase of apoptosis-linked gene-2 interacting protein $\mathrm{X}$ in areas of kainate-induced neurodegeneration. Neuroscience 123:887-895

42. Nixon RA (2007) Autophagy, amyloidogenesis and Alzheimer disease. J Cell Sci 120:4081-4091

43. Filimonenko M, Stuffers S, Raiborg C, Yamamoto A, Malerød L, Fisher EM, Isaacs A, Brech A, Stenmark H, Simonsen A (2007) Functional multivesicular bodies are required for autophagic clearance of protein aggregates associated with neurodegenerative disease. J Cell Biol 179:485-500

44. Parkinson N, Ince PG, Smith MO, Highley R, Skibinski G, Andersen PM, Morrison KE, Pall HS, Hardiman O, Collinge J, Shaw PJ, Fisher EM; MRC Proteomics in ALS Study; FReJA Consortium (2006) ALS phenotypes with mutations in CHMP2B (charged multivesicular body protein 2B). Neurology 67:1074-1077

45. Cox LE, Ferraiuolo L, Goodall EF, Heath PR, Higginbottom A, Mortiboys H, Hollinger HC, Hartley JA, Brockington A, Burness CE, Morrison KE, Wharton SB, Grierson AJ, Ince PG, Kirby J, Shaw PJ (2010) Mutations in CHMP2B in lower motor neuron predominant amyotrophic lateral sclerosis (ALS). PLoS One 5:e9872

46. Isaacs AM, Johannsen P, Holm I, Nielsen JE; FReJA consortium (2011) Frontotemporal dementia caused by CHMP2B 
mutations. Curr Alzheimer Res 8:246-251

47. Yamazaki Y, Takahashi T, Hiji M, Kurashige T, Izumi Y, Yamawaki T, Matsumoto M (2010) Immunopositivity for ESCRT-III subunit CHMP2B in granulovacuolar degeneration of neurons in the Alzheimer's disease hippocampus. Neurosci Lett 477:86-90

48. Kim BY, Olzmann JA, Barsh GS, Chin LS, Li L (2007) Spongiform neurodegeneration-associated E3 ligase Mahogunin ubiquitylates TSG101 and regulates endosomal trafficking. Mol Biol Cell 18:1129-1142

49. Jiao J, Sun K, Walker WP, Bagher P, Cota CD, Gunn TM (2009) Abnormal regulation of TSG101 in mice with spongiform neurodegeneration. Biochim Biophys Acta
1792:1027-1035

50. Reid E, Connell J, Edwards TL, Duley S, Brown SE, Sanderson CM (2005) The hereditary spastic paraplegia protein spastin interacts with the ESCRT-III complex-associated endosomal protein CHMP1B. Hum Mol Genet 14:19-38.

51. Lee JA, Gao FB (2008) ESCRT, autophagy, and frontotemporal dementia. BMB Rep 41:827-832

52. Lee JA, Gao FB (2009) Inhibition of autophagy induction delays neuronal cell loss caused by dysfunctional ESCRT-III in frontotemporal dementia. J Neurosci 29:8506-8511

53. Carlton JG, Agromayor M, Martin-Serrano J (2008) Differential requirements for Alix and ESCRT-III in cytokinesis and HIV-1 release. Proc Natl Acad Sci U S A 105:10541-10546 